\title{
EP-143
}

\section{Dose severity of acute cholecystitis related to postoperative visceral pain?}

\author{
Young II CHOI ${ }^{* 1}$, Ji Hoon JO', Hyung Hwan MOON', Hyung Joo CHUNG ${ }^{2}$, Dong Hoon SHIN' \\ 'Department of Surgery, Kosin University Gospel Hospital, Busan, Korea \\ ${ }^{2}$ Department of Anesthesiology and Pain Medicine, Kosin University Gospel Hospital, Busan, Korea
}

Introduction: The purpose of this study was to assess the correlations between postoperative visceral pain after laparoscopic cholecystectomy and the severity of acute cholecystitis (AC) using the Parkland grading scale (PGS).

Methods: A total of 138 patients with AC between 19 and 86 years of age classified according to PGS were assessed. All patients evaluated for postoperative visceral pain by measuring the use of analgesics over time in a patient-controlled analgesia (PCA) pump.

Results: High PGS grade was positively correlated with the amount of PCA during postoperative two hours $(p<0.05)$.

Conclusions: For the patient with AC in this study, correlations of postoperative visceral pain and severity of AC were positive. The PGS is helpful to control postoperative visceral pain differently depending on severity of AC. 